\title{
RESET
}

Recherches en sciences sociales sur Internet

$4 \mid 2015$

Les mondes du jeu

\section{Le jeu vidéo comme pratique discréditable}

Enquête sur les efforts de légitimation ordinaires des joueurs

Video games as discreditable practice. A study of gamers' everyday

legitimization efforts

\section{David Gerber}

\section{(2) OpenEdition}

Journals

Édition électronique

URL : http://journals.openedition.org/reset/452

DOI : 10.4000/reset.452

ISSN : 2264-6221

\section{Éditeur}

Association Recherches en sciences sociales sur Internet

\section{Référence électronique}

David Gerber, «Le jeu vidéo comme pratique discréditable », RESET [En ligne], 4 | 2015, mis en ligne le

21 décembre 2015, consulté le 19 avril 2019. URL : http://journals.openedition.org/reset/452 ; DOI :

$10.4000 /$ reset.452

Ce document a été généré automatiquement le 19 avril 2019.

(c) Association Recherches en sciences sociales sur Internet 


\title{
Le jeu vidéo comme pratique discréditable
}

\author{
Enquête sur les efforts de légitimation ordinaires des joueurs \\ Video games as discreditable practice. A study of gamers' everyday \\ legitimization efforts
}

David Gerber

\section{Introduction}

1 Cet article part du constat que les jeux vidéo occupent dans nos sociétés un statut ambigu de par leur position incertaine dans les hiérarchies culturelles d'une part, et de par les diverses conséquences indésirables dont on soupçonne leur pratique d'autre part. Si la valeur sociale et culturelle accordée au jeu vidéo constitue un objet d'actualité jusque dans les sciences sociales, il semble aussi nécessaire de se pencher sur la manière dont la question de la valeur du jeu vidéo se pose dans les vécus des joueurs, et comment ces derniers parviennent - ou non - à rendre leur pratique vidéoludique acceptable aux yeux de leur entourage familial et amical.

\section{Une légitimité ambiguë}

On peut en effet relever à un niveau macrosociologique des éléments contradictoires dans la valeur accordée aux jeux vidéo. D'un côté, on assiste à un processus de légitimation du jeu vidéo : des institutions productrices et reproductrices des valeurs culturelles légitimes (Bourdieu, 1979) semblent effectivement avoir érigé le jeu vidéo au rang d'objet culturel légitime (Ter Minassian, 2013). De l'autre, le média vidéoludique fait l'objet de controverses au sujet de ses impacts sur ceux qui le consomment, avant tout à propos de ses supposées propriétés addictives et de sa capacité à engendrer des comportements violents (Cover, 2006 ; Ferguson, 2008 ; Mauco, 2011). 
3 Du point de vue des institutions culturelles établies, le jeu vidéo semble jouir d'une attention de plus en plus bienveillante. D'abord au vu de l'intérêt que lui portent depuis quelque temps des musées d'art renommés ${ }^{1}$ ou des fondations nationales comme Pro Helvetia en Suisse, qui ont commencé à inclure les jeux vidéo dans le giron de leurs activités d'exposition et de promotion artistique. S'ajoute à cela l'attention, non dénuée d'enthousiasme, des médias généralistes (Gerber, 2010). La manière dont les médias cadrent le jeu vidéo s'apparente parfois à un processus d' «artification ${ }^{2}$ » (Shapiro \& Heinich, 2012) : le Figaro 3 par exemple a repris la formule du «dixième art » pour qualifier les œuvres vidéoludiques. S'il ne s'agit pas pour autant d'une forme culturelle highbrow ni tout à fait légitime, pour Jenkins (2006) ces formes de consécration rejoignent la valorisation par nos sociétés de la culture "geek », et par conséquent le jeu vidéo porterait de moins en moins de connotations négatives auparavant associées à l'électronique de loisir. Dans le monde scientifique également l'émergence des game studies ${ }^{4}$, en érigeant le jeu vidéo en objet d'étude digne d'attention scientifique, contribue à œuvrer dans le sens d'une légitimation partielle. Smuts (2005) et Wolf (2012) par exemple argumentent que le jeu vidéo n'a rien à envier à d'autres medias culturels et qu'il peut être le support d'une œuvre artistique. Enfin, on observe aussi des efforts de légitimation sur les sites web d'information vidéoludique et dans les forums dédiés aux jeux, comme en témoigne le titre d'un article du blog Gamasutra: «Video games are a legitimate form of cultural expression... right? $»^{5}$

4 Toutefois, comme le souligne ce même billet de blog, les jeux vidéo continuent à faire l'objet de controverses, parfois aussi qualifiées de "paniques morales» (Cohen, 1980 ; Cover, 2006). Dans les médias d'information comme dans les débats politiques, la pratique des loisirs numériques est soupçonnée de susciter des dépendances, de favoriser la violence ou l'isolement relationnel (Williams, 2003). Ces soupçons surgissent aussi dans le monde scientifique, où des études cherchent notamment à mesurer les « effets » des jeux sur écran. Parfois il s'agit d'effets considérés bénéfiques (Green \& Bavelier, 2003; Granic, Lobel \& Engels, 2013), mais plus souvent, on cherche à mesurer des influences sur les joueurs en termes d'agressivité (cf. Anderson \& Bushman, 2001; Ferguson, 2007) et d'addiction (Young, 1998 ; Gentile et al., 2011). Ces travaux font en retour l'objet de critiques pour leur alarmisme (Ferguson, 2008), pour leur modélisation réductrice de la réception des médias (Cunningham, Engelstätter \& Ward, 2011), ou encore pour leur conception unilatérale de la valeur des engagements sociaux (Dellwing, 2013). Les joueurs aussi participent parfois à la controverse : le traitement médiatique du jeu vidéo suscite régulièrement des positionnements défensifs de la part de blogueurs individuels ${ }^{6}$ allant jusqu'à des formes de mobilisations prolongées et collectives comme dans le cas du blog allemand Stigma Videospiele ou encore de l'association suisse Gamerights. ${ }^{7}$

5 Le jeu vidéo suscite donc de toutes parts des prises de position d'acteurs qui s'apparentent, en termes beckeriens, à des entrepreneurs de morale visant pour les uns à discréditer et pour les autres à valoriser le jeu vidéo, ce qui a pour résultat une incertitude quant au statut social et culturel du jeu vidéo. Mais l'ambiguïté est d'autant plus forte que deux choses distinctes sont en fait concernées : s'il semble bien que le jeu vidéo en tant qu'objet réussisse à accéder à un statut de bien culturel valorisé par les institutions légitimes, les pratiques, elles, demeurent sujettes à critique. Il semble donc qu'un processus de légitimation partielle soit en cours, mais qu'il s'accompagne d'une reconfiguration et d'un affinage des hiérarchies : comme le précise Berry (2012) à la suite de Bruno (1993), les différences de légitimité et d'acceptabilité semblent s'ériger non pas 
face au jeu vidéo dans son ensemble, mais parmi les jeux et les façons de jouer. Il devient ainsi important de s'intéresser aux pratiques vidéoludiques en contexte, afin de saisir les logiques sociales qui sous-tendent l'acceptabilité de ces pratiques.

\section{Etudier le jeu en contexte}

6 Par tradition, les travaux de game studies s'intéressent davantage aux jeux qu'à leurs usages par les joueurs. Parfois, les game studies s'intéressent empiriquement aux pratiques effectives et aux joueurs existants, mais le lecteur de sciences sociales peut rester sur sa faim, notamment devant le recours à une figure simplifiée du «joueur $»^{8}$ qui reste ainsi souvent une entité abstraite, une fonction du jeu (Aarseth, 2007). Certaines études vont toutefois au-delà de cette simplification en étudiant empiriquement les joueurs et leurs engagements. Lorsque les game studies s'intéressent en effet à la pratique vidéoludique se constituant ainsi en "play» studies (Triclot, 2013) - il devient possible de penser l'activité dans un cadre plus complexe que le strict « cercle magique » qui séparerait le jeu d'autres activités (voir à ce propos Zabban, 2012). L'étude des pratiques vidéoludiques peut s'inspirer tant de la sociologie des pratiques culturelles que des travaux sur les usages et des appropriations des objets de la communication (Jouët, 2000), en les envisageant sous l'angle de cadres de l'expérience (Fine, 1983 ; Berry, 2008) ou encore comme des formes sociales donnant lieu à des "styles" basés sur une variété de motivations, de relations sociales et d'écologies matérielles (Boutet, 2012).

7 Les play studies permettent de diverses manières de mettre en exergue l'ancrage social du sens que prend la pratique vidéoludique. Le sens du jeu pour les joueurs apparaît en effet lorsque les études rendent compte tant de la complexité sociale à l'intérieur des mondes ludiques qu'à l'extérieur, ce que les ethnographies (Taylor, 2006 ; Boutet, 2008 ; Nardi, 2010 ; Berry, 2012) et enquêtes démographiques par questionnaire (Williams, Yee \& Caplan, 2008 ; Berry, Coavoux \& Boutet, 2014) exemplifient bien. L'analyse des enjeux ordinaires liés à l'acceptabilité sociale du jeu vidéo s'inscrit dans une telle perspective de play studies. Comme le montrent d'autres travaux, il s'agit de tenir compte des ancrages relationnels des joueurs. L'étude des jeux vidéo des enfants dans l'espace domestique (Dajez \& Roucous, 2009a) par exemple, montre que les formes des pratiques répondent à des logiques de négociation des conditions du jeu et de construction du goût individuel. Dajez et Roucous soulignent en particulier le rôle essentiel que jouent les parents, la fratrie et les pairs. Chez les adultes aussi, malgré la disparition de l'autorité parentale, l'activité s'inscrit dans un ensemble de contraintes et d'incitations d'ordre social qui se donnent notamment à voir dans les relations entre le joueur et son entourage proche. Comme le suggèrent la sociologie des loisirs adolescents (Pasquier, 2005) ou encore les travaux anglophones sur le loisir (Kleiber \& Kelly, 1980 ; Raymore et al., 1999), le jeu vidéo dans les étapes de vie ultérieures est sujet à des problèmes de mise en adéquation avec des ressources, des sociabilités et des attentes de rôle certes différentes de celles des enfants mais non moins importantes. Tant les pratiques vidéoludiques des jeunes adultes (Thornham, 2009) que celles des personnes "plus âgées» (Quandt, Grueninger \& Wimmer, 2009) mettent les joueurs face à des contradictions et des conflits potentiels.

En définitive, le statut disputé et incertain du jeu vidéo au sein des controverses scientifiques et médiatiques nous invite à nous pencher sur la manière dont les acteurs se confrontent à des contraintes normatives et négocient les conditions d'acceptabilité de la pratique du jeu vidéo. L'article procède pour ce faire en deux temps : il énumère d'abord 
les façons dont la pratique vidéoludique se présente comme inacceptable aux joueurs et à leur entourage, puis propose une échelle des manières dont les joueurs composent avec voire agissent sur - l'acceptabilité de leurs pratiques ludiques. Ces positionnements sont présentés sur un continuum inspiré de la sociologie interactionniste, allant des formes des plus contraintes aux plus revendicatives. Sur cette base, nous réfuterons tant l'idée d'un stigmate du joueur inhérent à toute pratique vidéoludique que celle d'une légitimité systématique de la pratique du jeu vidéo.

Cet article s'appuie sur l'analyse de 28 entretiens biographiques avec des joueurs, récoltés dans le cadre d'une thèse de doctorat ${ }^{9}$. Les entretiens consistaient à faire raconter en détail son expérience avec les jeux vidéo depuis l'enfance. Le thème de l'acceptabilité des pratiques, s'il n'émergeait pas spontanément, était abordé à travers une série de questions plus focalisées: Dans quelle mesure est-ce que l'entourage familial et professionnel connaît ses jeux et pratiques? Avec qui parle-t-on, ou non, de jeux vidéo ? Et quelle opinion a-t-on des débats sur le jeu vidéo qui émergent dans les médias, en particulier sur la violence et l'addiction? Les entretiens ont été enregistrés, retranscrits et soumis à une analyse de contenu qualitative qui a permis d'identifier et de coder les passages concernant les enjeux de l'acceptabilité du jeu. Cette analyse transversale était complétée par les informations biographiques et les notes d'observation afin de contextualiser les récits de pratiques et positionnements exprimés.

\section{Les expressions de l'inacceptabilité}

10 Si donc le jeu vidéo est une pratique au statut incertain, sous quelles formes l'illégitimité et l'inacceptabilité se manifestent-elles? Comment sont-elles perçues par les joueurs? Dans la mesure où les données de l'enquête ne contiennent pas directement les discours de l'entourage relationnel des joueurs, mais que ces réprobations sont appréhendées par le biais des récits des joueurs, leurs arguments précis ne sont souvent pas distinctement observables. Les récits des joueurs portent plutôt sur des situations où des jugements sont émis par des acteurs qui sont eux plus aisément saisissables, et qui condamnent explicitement ou implicitement un ou plusieurs aspects de la pratique vidéoludique. C'est pourquoi l'inacceptabilité est présentée ici en fonction des personnes et des situations au travers desquelles elle se manifeste.

11 Comme l'on peut s'y attendre, ces manifestations émanent en premier lieu des parents, comme la mère de Kriek ( $\mathrm{H}, 28$ ans, employé de banque) qui n'aimait pas qu'il « tue des gens sur le PC» du salon, ni qu'il tape frénétiquement sur le clavier en lors de ses tournois de Tetris en ligne. Kriek raconte la pratique secrète du jeu vidéo adoptée à l'époque de sa première année d'université, qu'il a par ailleurs décidé d'abandonner, n'y portant pas suffisamment d'intérêt :

Je partais de chez moi avant ma mère, prendre le train, je faisais le tour du bloc à pied, ma mère partait entre-temps, je rentrais, j'allumais le PC, donc il était $8 \mathrm{~h}$ du mat, je jouais, ma mère rentrait à $16 \mathrm{~h} 30$. À $16 \mathrm{~h}$ j'éteignais le $\mathrm{PC}$, je montais, ma mère revenait, ça a été la journée? Ouais on a eu trois heures de cours, je vais allumer le PC je vais faire une petite partie. Voilà. (Kriek H, 28 ans, employé de banque).

Ces manœuvres montrent que Kriek opérait une distinction entre une pratique acceptable aux yeux de sa mère (la "petite partie» en fin de journée) et celle, inappropriée, consistant à y passer la journée. Il ne s'agit pas là d'une question 
d'illégitimité culturelle, mais bien plutôt de l'inacceptabilité du fait de jouer plutôt que d'aller en cours. À l'instar de cet exemple, le principal jugement parental que relatent les récits des joueurs porte sur l'agencement des priorités entre activités, ainsi que sur les modalités temporelles de la pratique : durée, fréquence. La citation de ce joueur indique que même dans un cas moins extrême en termes d'infractions aux attentes parentales, la source de désapprobation se trouve davantage dans la gestion du temps que dans la «nature » de la pratique :

Quand j'étais encore chez ma mère bon ben des fois ça la saoulait un peu, genre elle m'appelait pour manger, $j$ 'dis ouais c'est bon j'arrive dans dix minutes quoi. Et bon je venais qu'une demi-heure après [rires] (...) Des fois elle était juste saoulée que je joue, bon ben voilà quoi. Mais non j'ai pas eu comme reproche euh, t'es drogué du jeu ou ce genre de chose. (Onyx, H, 27 ans, étudiant en droit).

Quelques instances de jugements portant plus clairement sur une illégitimité culturelle se trouvent tout de même, par exemple chez les parents de Malik (H, 29 ans, employé de banque) dont ce dernier rapporte les reproches entendus lors de son adolescence: «tu perds tout ton temps, faut que tu bosses" plutôt que de jouer à des jeux qui « rendent stupide ». Là encore, le jugement de la pratique est lié à la gestion du temps, mais la qualification des jeux rendant "stupide» constitue un jugement de valeur fortement négatif en termes de hiérarchie culturelle, si l'on pense à la déclinaison des dichotomies entre culture highbrow et lowbrow de L. Levine (Lahire, 2004 : 78). Une piste d'explication à envisager ici est que contrairement aux interviewés précédents, Malik est d'origine sociale supérieure, avec des parents qui sont ainsi susceptibles d'avoir des attentes plus « légitimistes » quant aux pratiques de loisir de leurs enfants. Une seconde piste, complémentaire, à envisager réside dans le fait que Malik a dû redoubler une année de baccalauréat pour avoir trop joué à un jeu de rôle en ligne, en se servant par ailleurs de la carte de crédit de son père à l'insu de ce dernier, suite à quoi il a cessé toute pratique du jeu vidéo à proprement parler. Il est donc vraisemblable que le caractère abrutissant prêté aux jeux renvoie à cet échec scolaire.

Au final, parmi nos données, l'indication la plus tangible d'un pur et simple rejet du jeu vidéo subi par les joueurs se trouve dans les jugements émanant des camarades de classe de ce même Malik, qui se souvient qu'à l'âge du CM2 environ « les filles elles disaient c'est des petits jeux de gamin et tout [rires] donc au final c'était pas très bien vu »; jugement qui s'appliquait tant aux jeux vidéo qu'aux jeux de figurines sur table, et relègue la pratique du jeu vidéo au statut d'activité enfantine. Les travaux sur les objets culturels de l'enfance de Dajez et Roucous (2009b) montrent bien que l'élaboration des goûts des enfants passe par des conceptions du bon âge pour chaque pratique, où les jeux pour les plus petits font figure de repoussoir. Par ailleurs, les différences de goûts sont fortement genrées à l'adolescence, y compris au sujet du jeu vidéo, comme l'indiquent les recherches de D. Pasquier sur les lycéens (Pasquier, 2005, 2010). Si ce jugement émanant des filles à l'adolescence peut avoir contribué au rapport "coupable» que Malik entretient avec les jeux vidéo, nous n'avons pas rencontré chez les autres joueurs de mentions du même phénomène. Peut-être parce que, comme le souligne avec insistance D. Pasquier, la forte différenciation s'accompagne d'une tendance à la hiérarchisation des goûts en faveur de ceux des garçons. En revanche la non-congruence des goûts masculins et féminins pour le jeu se manifeste encore plus tard dans les vies des joueurs.

Les parents et les camarades de classe constituent les principaux acteurs dont les évaluations de légitimité comptent pour les joueurs adolescents. Nos enquêtés cependant 
se situent souvent, au moment de l'entretien, dans des étapes de vie plus avancées. Chez les joueurs en couple, le conjoint constitue une personne dont le jugement de la pratique vidéoludique est fréquemment mentionné. Conformément à un cliché répandu, les joueurs mentionnent des " problèmes » avec leur femme ou des tensions avec leur petiteamie (ou, plus rarement, leur petit-ami). En creusant la question, Samuel (H, 37 ans, informaticien) précise toutefois un détail qui amène une nuance intéressante : «Je n'ai jamais été confronté à quelque chose de négatif. De l'indifférence [de la part de ma femme], oui, mais un rejet jamais. » Cette indifférence que décrit Samuel consiste en ce que sa femme ne s'y intéresse que « le temps que je lui parle, mais effectivement elle s'en tape totalement ». Il a " essayé un petit peu sans trop y croire » de l'initier à ses jeux, sans succès, et se l'explique par le fait qu' « elle est pas du tout dans le même univers que moi. On a beaucoup de points communs mais pas celui-là. » On observe en effet que les joueurs doivent composer avec des différences de goûts, sans que cela n'implique clairement l'expression de jugements d'infériorité en valeur. À y regarder de plus près, au cœur de ces tensions se trouve davantage un enjeu de gestion du temps de jeu qu'une question de valeur culturelle. Ainsi la question au cœur des limites à l'acceptabilité du jeu, c'est le plus souvent le temps de jeu versus le temps passé avec la famille ou d'autres proches. À l'exemple de Samuel on peut ajouter celui de Melek qui raconte des «reproches » émis par sa petite amie :

Par exemple le soir j'avais un match [dans son jeu de tir en ligne] et qu'on parlait sur MSN à l'époque (...) et que j'répondais pas pendant quarante minutes, après j'me faisais engueuler, ouais tu pourrais m'répondre! (Melek, H, 20 ans, webdesigner indépendant).

Notons encore que les joueurs ne sont pas unilatéralement dans un rapport coupable à leur activité et peuvent, du moins ponctuellement, recevoir le soutien de leur entourage. Parfois les proches apprennent à apprécier l'aspect « social » des amitiés entretenues via le jeu. Aliss (F, 30 ans, informaticienne de gestion), joueuse de jeux de rôle en ligne a intégré son conjoint dans les relations amicales de son clan, si bien qu'il l'accompagne désormais lorsqu'elle se rend aux rencontres «IRL $»^{10}$, même si au début cela lui a «fait bizarre quand elle est allé voir son pote dans le sud de la France qu'elle avait jamais vu ». Parfois les proches valorisent et soutiennent activement les engagements vidéoludiques comme ils le feraient pour un engagement associatif ou sportif par exemple. Baraka (H, 25 ans, étudiant BTS), qui habite avec son père raconte que ce dernier " est à fond ", à savoir que les jours de match avec son équipe de FPS ${ }^{11}$, il avance l'heure du repas et lui demande des nouvelles quant aux résultats des compétitions. Ce type d'intérêt rapproché pour certains aspects jeu apparaît occasionnellement dans nos données, et rejoint les précédents indices pointant qu'il n'y a pas de condamnation systématique du jeu. Les parents, la fratrie ou la famille plus large sont souvent ceux qui offrent des jeux ou des consoles, et, particulièrement dans le cas des joueurs sans revenus, mettent à disposition leur PC et la connexion à internet et payent les éventuels abonnements aux jeux en ligne ; ceci même s'ils ne perçoivent pas le jeu comme une activité culturelle respectable.

Une autre situation, où la réprobation ne vient cette fois pas de l'entourage immédiat, apparait dans les entretiens: le traitement par les médias d'information des effets néfastes du jeu peut lui aussi être perçu comme une critique directe à leur loisir. Les joueurs relèvent à ce titre surtout l'association faite entre violence physique et violence vidéoludique.

Évidemment il y en a toujours qui diront les jeux vidéo c'est mal, c'est pas bien et tout, preuve dernier exemple la tuerie qui a eu lieu avec les gamins qui se sont fait 
descendre l'autre jour, quand tu regardes les titres des articles, y a toujours une ligne qui dit qu'ils jouaient aux jeux vidéo les gamins. Toujours. Sans qu'on dise ce que ça vient faire là. C'est systématiquement ainsi (Samuel, H, 37 ans, informaticien). dans la mesure où eux-mêmes n'en ressortent pas plus violents. Mais contrairement à la dénonciation du discours médiatique et la défense passionnée du jeu auxquels on pourrait s'attendre, les enquêtés se révèlent souvent nuancés sur ce thème. Ils sont même prêts à reconnaitre que certains individus peuvent être incapables de faire la différence entre réel et fictionnel. Comme le résume Lino:

Ouais, est-ce que c'est le fait qu'ils y aient joué qui les a rendus comme ça ou est-ce qu'ils étaient déjà comme ça avant d'y jouer (...) Mais non, moi non j'm'en fous [rires] (Lino, H, 24 ans, ouvrier intérim).

À l'instar de Lino, les joueurs interrogés ne se sentent généralement pas investis d'une mission de défense des jeux, sauf quelques exceptions. Les joueurs enquêtés tendent même à reconnaître une vraie pertinence aux interrogations à propos de l'addiction qu'induisent peut-être les jeux vidéo, comme on le verra par la suite.

'est ce qui nous amène à énumérer un dernier acteur émetteur de jugements. Il s'agit des joueurs eux-mêmes, qui énoncent des jugements de valeur sur le jeu vidéo en mobilisant des classements hiérarchiques des types de jeux et en catégorisant les autres joueurs et leurs rapports au jeu. Muriel, qui joue occasionnellement, identifie une valeur négative particulièrement liée aux jeux en ligne :

Je me limite, enfin je trouve que c'est un peu une drogue en fait les jeux vidéo. J'ai aussi d'autres amis qui me parlent de World of Warcraft, enfin tous ces jeux je les vois pris dedans, et puis je me dis, c'est quand même un peu antisocial (Muriel, F, 27 ans, assistante administrative).

Elle rejoint d'autres joueurs qui recourent au qualificatif de «drogue » pour parler de ce type de jeux. Dans les catégorisations qu'opèrent les joueurs, on se rapproche de l'idée d'un stigmate avec le qualificatif de nolife. Similairement à la catégorie de geek - lorsque celle-ci est utilisée péjorativement - celle de nolife est rarement endossée par les joueurs eux-mêmes, si ce n'est dans des expressions comme "faire le nolife ", jouer " comme un gros geek». Chai (H, 26 ans, technicien) en racontant son rapport à Diablo $2^{12}$, qu'il juge inintéressant mais addictif et auquel il a joué trois mois, dit « je faisais le nolife dessus, mais ça m'a vraiment pas trop marqué. » Le terme sert de figure repoussoir, servant à qualifier quelqu'un (ou une partie de soi ${ }^{13}$ ) dont on cherche à se distancier, parce le nolife passe un nombre d'heures excessif à jouer et n'a pas d'autres intérêts ni responsabilités ; il n'a en somme pas de « vraie » vie. De même lorsque Kriek relate la première rencontre en face à face avec son équipe il a constaté que

On est pas une équipe de gros geeks quoi. Quand on était à [lieu de la rencontre] on n'était pas une bande de gros pleins de boutons, puants, qui ne parlent que de ça quoi (Kriek, H, 28 ans, employé de banque).

Il est intéressant de noter que l'usage le plus stigmatisant de ces termes se rencontre in fine dans la bouche des joueurs eux-mêmes. Ces recours aux stéréotypes peuvent s'interpréter comme une anticipation et une mise à distance du stigmate qui passe par la désignation d'un coupable autre que soi, mécanisme qu'ont déjà souligné les recherches anthropologique sur la réaction sociale au risque (Joffe, 1999).

Il apparaît ci-dessus que l'inacceptabilité de la pratique vidéoludique est de nature variable, plus complexe qu'une catégorisation du jeu vidéo comme globalement 
sanctionné par un «stigmate du joueur». L'expression d'une illégitimité culturelle au sens "classique ", à savoir que le jeu comme forme culturelle serait de valeur inférieure par rapport à d'autres formes culturelles, apparaît certes au travers de sa qualification d'activité enfantine (les "petits jeux pour gamins»), du renvoi à son caractère improductif (jeux «inutiles», "perte de temps») ou abrutissant (jeux qui «rendent stupide »). Mais ces jugements ne portent que rarement sur les jeux vidéo en tant que catégorie d'ensemble. Comme le souligne Berry (2012), un des signes du processus de légitimation culturelle en cours est la hiérarchisation non pas du jeu vidéo en général, mais de titres et de genres en particulier, et de pratiques plus ou moins légitimes. Les jugements négatifs sur le jeu observés portent sur un ensemble d'aspects qu'il nous semble possible de partager en deux types: la forme et les contenus d'une part (dimension guerrière et violence représentée typiquement dans les jeux de tir ; nature enfantine de "petits " jeux; simplicité et manque de profondeur de certains jeux de navigateur ou de smartphone; ...) et l'engagement dans les jeux d'autre part (importance donnée aux jeux par rapport à d'autres activités et relations ; gestion de la durée, de la fréquence et choix des moments de jeu ...) Cette distinction est utile parce qu'elle permet de penser la légitimité au niveau des vécus individuels sans imposer a priori des catégories hiérarchiques sanctionnant la valeur culturelle à l'échelle de toute la société, et permet de souligner l'importance plus saillante des questions normatives autour des modalités de la pratique, et en particulier de la gestion temporelle.

24 A travers les différents exemples présentés, il apparaît que le jeu n'est pas d'abord inacceptable, mais qu'il peut constituer une pratique appropriée, du moment qu'elle reste "à sa place", à savoir qu'elle n'empiète pas sur d'autres activités - considérées prioritaires - de la vie quotidienne, scolaire, professionnelle, et familiale. Si donc la place " secondaire » des activités ludiques semble faire consensus, les définitions de cette place, et de ce qui constitue un débordement font l'objet de négociations et de tensions. Ces tensions peuvent aussi être comprises, comme l'argumente Dellwing (2013) comme un problème de gestion de l'attention. Ce problème s'exprime par des conflits autour des attentes de présence à la relation versus l'absence qu'implique l'engagement dans des relations ludiques perçues comme artificielles et addictives. Cependant, les joueurs partagent et prononcent parfois eux-mêmes le verdict sur l'excès de jeu. Gauthier (20 ans, étudiant de bachelor), qui dit s'être "perdu dans le jeu " pendant une période, explique que son père désapprouve sa pratique, mais que lorsqu'il « joue raisonnablement, ça va, il supporte mieux ». Il devient plus difficile de considérer le point de vue du père comme purement «externe» d'autant plus que ce dernier est informaticien et joue aussi occasionnellement à l'ordinateur. Gauthier a cessé de jouer aux jeux vidéo, mais tel n'est pas le cas d'autres enquêtés, qui ont élaboré diverses manières de composer avec ces tensions, ce que nous développerons plus avant à présent.

\section{Positionnements face à l'inacceptabilité : de l'évitement à la revendication}

Dans ce qui précède, on aura deviné que les joueurs, confrontés au à la possibilité du discrédit, se trouvent amenés à se positionner par rapport à leur pratique (Bamberg, 1997). Nous allons détailler à présent les manières dont les joueurs évitent, négocient ou contestent l'inacceptabilité de leur pratique. Ce sont des formes de «légitimation » non pas en termes de légitimité culturelle, mais dans le sens où elles permettent de rendre 
acceptable son engagement ludique grâce à des discours et des pratiques permettant de le " cadrer» (Goffman, 1986) comme approprié. Les modalités de ces efforts sont présentées en allant des plus « conciliantes » aux plus « ambitieuses » : l'évitement du jugement par la pratique secrète du jeu ou par l'adaptation des modalités de la pratique, la relativisation et le fait de nuancer l'inacceptabilité, la mise en valeur des propriétés valorisées du jeu, et finalement les projets de changement des échelles de valeur auxquelles sont jaugées les pratiques vidéoludiques. Les éléments de cette typologie ne sont pas mutuellement exclusifs et ne se trouvent pas nécessairement à l'état pur empiriquement, mais ils nous paraissent suffisamment récurrents et contrastés pour être présentés de la sorte.

\section{Éviter le stigmate: occultations et adaptations de la pratique}

Si l'on n'observe pas une stigmatisation généralisée des joueurs dans les récits de nos enquêtés, il existe en revanche dans la variété des vécus liés à la pratique vidéoludique des formes de pratiques et types de jeux plus ou moins perçus comme acceptables et où la réflexion goffmannienne sur le stigmate et le contrôle de l'information deviennent utiles (Goffman, 1975). Les joueurs, même avec de forts degrés d'engagement dans le jeu tendent à éviter d'en parler autour d'eux. Par exemple ce joueur qui estime irrecevable l'expression de sa passion vidéoludique auprès des personnes dont il ne connaît pas déjà un intérêt pour les jeux :

C'est pas quelque chose que je vais amener à un repas de famille, ou que je vais amener avec les groupes d'amis à qui j'en ai jamais parlé jusque-là. (...) c'est quelque chose que je garde pour moi en général (...) Peut-être que je pensais que ça les intéresserait pas forcément. En tout cas j'me dis que c'est souvent le cas (Samuel, $\mathrm{H}, 37$ ans, informaticien).

De manière similaire, Chai évite d'afficher son engagement dans les jeux de tir en ligne, dont il perçoit des limites à l'acceptabilité :

$\mathrm{Y}$ a pas tout le monde qui sait, à part ma famille proche, ma famille mes parents et mes frères, $\mathrm{y}$ a personne d'autre vraiment qui sait que je joue régulièrement à ces jeux. Ils savent plus que je suis dans le jeu de football [non-vidéoludique] que le FPS. $C^{\prime}$ est pas quelque chose que j'affiche en grand là en large (...) voilà c'est pas vraiment une gloire de tuer des gens dans un jeu vidéo, c'est pour ça que je l'affiche pas (Chai, H, 26 ans, technicien).

Les propos de Chai pointent clairement le fait que tuer des avatars ennemis est " discréditable », ce qui concerne bien des jeux, et en particulier dans le genre du FPS. La violence ludique peut se révéler en effet comme une source de discrédit particulièrement dans l'anticipation qu'en font les joueurs. En effet, outre les exemples cités ici, la plupart des joueurs disent n'avoir jamais concrètement subi de la part d'autrui de jugements négatifs à l'encontre de leur hobby, mais force est de constater qu'ils évitent avec soin de s'exposer à cette possibilité.

Si les causes de cette anticipation ne sont d'ordinaire pas des expériences de stigmatisation vécues, comment peuvent-elles être interprétées? Il semble que l'enjeu dépasse à nouveau la question d'une légitimité culturelle. De manière générale, rares sont les joueurs interrogés qui disent communiquer, même avec leur famille proche, à propos de ce qu'ils font précisément dans leurs jeux. Lino (H, 24 ans, ouvrier intérim) par exemple jouit d'une bonne réputation au sein de la communauté de son jeu FPS pour avoir fourni des contributions dont la création de plusieurs cartes de trickjumping ${ }^{14}$ et la mise en ligne de vidéos montrant divers usages détournés du programme, au point où il a 
été recruté dans l'équipe bénévole de développement du jeu pour participer à la conception des cartes officielles. Cependant, cette reconnaissance ne s'étend pas au-delà de la communauté ludique. Ses parents, avec qui il habite, ne connaissent pas la nature exacte de ses activités ludiques : «Bon ils trouvent que je passe trop de temps sur l'ordi, voilà [rires]. Ils savent pas vraiment comment j'suis impliqué dedans. » De même pour Sirius (H, 20 ans, BTS en emploi) : « C'est difficile de le faire comprendre [l'intérêt pour le jeu]. En général t'en parles pas trop ». Il apparait qu'au fond, l'anticipation d'un jugement négatif de la part de l'entourage participe plus généralement d'une difficulté à " faire comprendre » à l'extérieur l'intérêt porté au jeu, ce que corrobore le positionnement de joueurs comme Sirius, impliqués dans ce que l'on pourrait qualifier des projets collectifs de légitimation du jeu vidéo, positionnements qui seront détaillés dans les formes de légitimation plus loin.

L'incompréhension de la nature de l'activité ludique vue de l'extérieur est selon certains auteurs à l'origine de la définition de la pratique comme une addiction (Wendling, 2006; Dellwing, 2013). De la même manière, au cœur des problèmes de légitimation pour les joueurs semble donc résider le fait qu'il est difficile aux proches non-impliqués dans le jeu de savoir ce qu'il s'y passe précisément si ce n'est que le joueur "passe trop de temps devant l'ordi ", voire joue à des jeux enfantins, abrutissants ou encore violents. De ce fait, le cadre d'interprétation appliqué au jeu, que ce soit par les pratiques ou les discours, est crucial pour la possibilité de jouer légitimement. L'exemple de Kriek ci-dessus est une des manières de définir le cadre par ce que Goffman appelle la « fabrication » (Goffman, 1986 : 83) en cachant sa pratique. Il s'avère cependant que cette manipulation n'est pas toujours possible, d'autant moins à long terme. Tôt ou tard, les joueurs interrogés se positionnent tant face aux difficultés de compréhension d'autrui qu'aux jugements sur la valeur des jeux et de leur pratique. La possibilité la plus radicale est vraisemblablement l'interruption de toute pratique du jeu vidéo. Parmi les joueurs ayant cessé leur pratique ludique, Malik, suite à un engagement assidu dans plusieurs jeux dont un jeu de rôle en ligne, raconte s'être interdit de jouer afin d'éviter de «foirer » sa vie professionnelle et académique.

J'ai gardé un peu cette idée d'interdit. Si je veux pas foirer mon diplôme à [école de gestion], j'sais pas, je sais que c'est un peu cliché, mais quand même cette idée que c'est- j'sais pas comment dire, pour bien utiliser mon temps il faut que je fasse ceci cela et puis les jeux vidéo je les vois encore un peu comme ça donc voilà (Malik, $\mathrm{H}$, 29 ans, employé de banque). adhère à l'idée que le temps serait mal investi dans le jeu, son rapport aux jeux reste ambivalent dans le sens où il s'interdit de jouer, tout en alimentant sa passion pour les jeux. En effet, il lit des livres portant sur les univers fictionnels des jeux et regarde régulièrement sur YouTube les vidéos des séquences animées tirées des jeux qui l'intéressent. D'un côté il adhère à l'idée que jouer est une "perte de temps ", mais en même temps trouve cela "dommage », et s'en explique en invoquant un «sentiment de culpabilité » et une sensation « de vide » après avoir passé ses vacances à jouer à un jeu. La culpabilité ressentie indique qu'il perçoit que le jeu est peu valorisé par son entourage, et qu'il accepte dans une certaine mesure ce jugement; mais cette acceptation seule ne suffirait vraisemblablement à le pousser à arrêter de jouer puisqu'il maintient une forme d'engagement vidéoludique en vivant sa "passion " pour les jeux sans y jouer. Face à ce qu'on pourrait appeler une incertitude normative sur le statut de la pratique vidéoludique, il semble donc que c'est en conjonction avec le second aspect cité 
qu'émerge une raison de cesser de jouer : ce sont les expériences de perte de contrôle sur sa pratique, qui se soldent par un sentiment de "vide », qu'il explique ainsi : «J'veux dire j'avais l'impression que les autres s'étaient amusés et puis moi j'avais passé mon temps à Starcraft. » Cette idée de perte se retrouve chez Gauthier qui a arrêté de jouer à son jeu de rôle suite à son constat que son expérience du jeu n'avait aucune valeur au-dehors :

Il y a aucune valorisation de savoir... moi, tout ce que je savais, toutes les connaissances que j'avais acquises c'était des connaissances qui valaient que sur un jeu. Je savais les astuces pour gagner beaucoup de PO [points d'or], des astuces pour grimper vite d'expérience, des trucs pour battre telle ou telle classe, mais tout ça ça sert à rien si... ça vaut zéro dans la vie réelle. Et donc j'me disais, si j'fais pas autre chose, euh.. ouais, j'vais être... perdu! (Gauthier, 20 ans, étudiant de bachelor).

Ces cas incarnent les formes d'adaptation les plus extrêmes, leur expérience s'étant soldée par un arrêt de la pratique du jeu. Plus fréquemment, les joueurs ont pu juger l'activité vidéoludique potentiellement inadéquate sans pour autant l'interrompre totalement. Samuel (H, 37 ans, informaticien), sur le point de devenir père, décrit les «terrains d'entente » que sa femme et lui ont dû trouver pour maintenir un rapport au jeu acceptable. Il s' " accorde des moments après le boulot, au plus une petite heure », et le week-end «à partir du moment où y a pas de trucs définis, ça va». Aliss (F, 30 ans, informaticienne de gestion), elle, a mis un terme à sa pratique compétitive en ligne, mais maintient aussi une pratique vidéoludique régulière en jouant de manière plus casual ${ }^{15}$ : « maintenant je me connecte le samedi matin et le dimanche matin pendant que j'ai mon ami qui dort. » Elle se réserve parfois une semaine de vacances pour "geeker » en se replongeant dans son jeu préféré comme auparavant. Baraka (H, 25 ans, étudiant BTS), qui a reçu des reproches de ses petites-amies à propos du temps passé à jouer, précise que «j'les lâche pas les weekends tout ça ». De la même manière qu'il souligne l'adaptation aux attentes des relations amoureuses, il relativise l'importance de la pratique du jeu en rapport aux relations amicales : «S'il y a une soirée avec des potes et un match sur [le jeu FPS], j'vais aller à la soirée avec mes potes quoi ». Pour ces raisons, son équipe de FPS a décidé de ne pas planifier de compétition les soirs de week-end.

Si les ajustements nécessaires au maintien d'une pratique acceptable sont fréquents, ils ne vont pas de soi. Les joueurs développent un autocontrôle dont l'acquisition peut constituer un processus d'équilibrage délicat, et les joueurs ayant abandonné entièrement la pratique vidéoludique ont vraisemblablement entamé un tel processus d'apprentissage sans réussir à « trouver un équilibre » comme les joueurs cités ici :

Mais j'ai trouvé un équilibre de vie sans devoir me forcer. J'ai suffisamment effectivement d'emprise sur moi pour arriver à le faire sans que ça me pose un problème. Puis c'est toujours un plaisir de sortir avec des amis, de boire un coup, en fait je dirais que maintenant effectivement c'est du 50-50 (Samuel, H, 37 ans, informaticien).

Je fais quand même la part des choses entre les deux même si c'est pas forcément facile d'avoir deux vies entre guillemets (Melek, H, 20 ans, webdesigner indépendant).

On peut faire l'hypothèse que cet autocontrôle est lié à la capacité des joueurs à s'adapter à des attentes de rôle. Dans les cas d'Aliss et Samuel, ces rôles sont directement liés à de nouvelles étapes dans leurs parcours de vie. L'intériorisation des attentes de rôle et la reproduction des normes liées aux étapes de vie ont par ailleurs depuis longtemps été soulignés par la sociologie des parcours de vie (Neugarten, Moore \& Lowe 1965). 


\section{Minorer le stigmate: nuancement et déplacement}

Les joueurs ne doivent donc pas nécessairement abandonner leur pratique pour être en conformité avec les diverses attentes normatives. De plus, il n'y a pas toujours besoin d'adapter sa pratique, car elle est peut être perçue comme suffisamment proche de ce qui est «normal ». Lorsque les risques d'inacceptabilité sont réduits, des arguments peuvent être adressés aux jugements normatifs, qu'ils soient effectifs ou anticipés.

Une première façon de concevoir et de communiquer le sens de sa pratique vidéoludique, est de présenter cette dernière comme relevant d'un droit à disposer à sa guise de son temps « libre ». C'est ce à quoi font par exemple référence les joueurs quand ils parlent de s'accorder un moment de détente après le travail ou de "se faire plaisir » en contraste avec des activités moins plaisantes, plus astreignantes. On peut aussi regrouper sous ce type le fait d'établir une équivalence entre jeu vidéo et autres pratiques plus établies. Par ailleurs, les joueurs précisent souvent que le jeu vidéo constitue un loisir parmi d'autres, comme le sport, les sorties ou la lecture, quand bien même le jeu en est le plus assidument pratiqué chez les joueurs les plus engagés: «En soi c'est pas pire que de passer son temps devant la télé » (Lino, H, 24 ans, ouvrier intérim).

Ce recours à l'argument de la banalité donne lieu à plusieurs manières de contester l'inacceptabilité de la pratique vidéoludique dans les discours des joueurs. Un joueur cité plus tôt disait n'en avoir rien à faire de la légitimité de sa pratique, ce à quoi fait écho la position d'Onyx, qui fournit une piste explicative par la relativisation de la place du jeu vidéo : «j'estime que ça prend pas une place énorme dans ma vie, et j'ai pas besoin d'en parler » (Onyx, H, 27 ans, étudiant en droit). Cependant, au vu des tensions que vivent les joueurs, surtout ceux fortement engagés, une seconde façon de cadrer le jeu consiste à le définir comme une passion ou un loisir, qui néanmoins "se gère " au même titre que d'autres activités et n'empêche pas par ailleurs un emploi à plein temps :

Ben je bosse des semaines de quarante heures, c'est une passion donc je fais ça comme une passion quoi. Comme toi tu fais diverses choses, diverses activités, $j$ 'ai aussi fait du football américain à l'époque donc ça se gère comme une activité (Sirius, H, 20 ans, BTS en emploi).

Les joueurs peuvent donc s'appuyer sur leurs réussites en termes d'adaptations de la pratique décrites plus haut et de leur capacité à remplir d'autres engagements comme le travail. L'on peut avancer aussi que le fait de passer du temps sur un ordinateur et sur Internet est devenu courant et qu'il n'y a plus de grandes différences entre joueurs et non-joueurs à cet égard :

Mais en tout cas voilà, internet comme ça devient vraiment de plus en plus grand public avec le milliard de possibilités que ça donne, eh bien j'me sens plus aussi, comment dire, marginal qu'avant. $\mathrm{Y}$ a pas que moi y a d'autres gens aussi qui passent beaucoup de temps sur l'ordi (Gauthier, 20 ans, étudiant de bachelor).

Le phénomène de généralisation mentionné ici s'applique aussi aux jeux vidéo, ainsi les joueurs peuvent invalider les jugements de valeur portant sur les jeux dans leur ensemble en invoquant la diversité croissante des jeux et des façons de jouer du jeu vidéo :

Il y en a tellement pour tous les goûts, que... ça serait très très réducteur de mettre tous les jeux vidéo dans le même panier (...) on peut plus, c'est devenu trop large maintenant pour qu'on puisse [les condamner] (Samuel, H, 37 ans, informaticien).

Et dans la même optique, Samuel mentionne la diversité croissante des publics du jeu vidéo qui contribue à la banalisation. Employé dans une institution médicale, il explique 
qu'il y a «beaucoup de médecins finalement » avec qui il parle jeux vidéo : «j'en ai un, là, qui me parlait de Black Ops [jeu de tir en vue subjective] qui me disait que je devais l'acheter et tout. » Parmi ses collègues joueurs, il relève le fait que certains ont le statut de médecin, précisément semble-t-il parce que le statut d'informaticiens ou d'autres employés n'a pas le même impact.

41 L'inacceptabilité potentielle est nuancée par la banalité de la pratique du jeu vidéo lorsque les joueurs invoquent le fait que « c'est un hobby comme un autre». Mais avec la diversité des jeux et des publics apparaît aussi une distinction interne qui suggère qu'il subsiste des hiérarchies parmi la variété des jeux. Samuel complète d'ailleurs sa position en remarquant qu' « après tout dépend à quoi tu joues ». Introduire des distinctions entre les jeux revient à souligner certaines propriétés supérieures en valeur et peut de ce fait être considéré comme un effort implicite de légitimation. Opposer les jeux « indépendants » aux jeux de grands éditeurs intéressés par les bénéfices des ventes; des jeux «riches" esthétiquement ou en contenu par opposition à des produits sans profondeur ou de mauvais goût; des jeux « intelligents » qui constituent d'une certaine façon une activité enrichissante pour l'esprit à des jeux superficiels; ou encore des jeux "novateurs" aux jeux conçus sans inventivité. Ces oppositions supposent une connaissance, une culture vidéoludique qui n'est pas présente chez tous les joueurs, car elle suppose une carrière ludique bien fournie, avec ce que cela implique de possession de matériel et de logiciels, et semble être le fait de joueurs occupant déjà un emploi plutôt que les joueurs adolescents et/ou étudiants.

Il s'agit d'un mode de légitimation par contraste à un objet moins légitime; un rapprochement avec les mécanismes de la distinction culturelle décrits par les études sur la légitimité culturelle devient ici tangible. Par exemple Malik introduit une hiérarchisation illustrant quelques-unes des oppositions de valeurs citées ci-dessus :

Avec les jeux de rôle il y a quand même tout un aspect je sais pas exactement comment appeler ça, c'est vraiment ouais toute la construction d'un monde, avec des personnages des compétences etc. Et puis c'est quand même un peu comme du théâtre, comme un jeu de société et tout et tout donc effectivement quoi c'est vrai que, parce que bon si je résume Diablo au final c'est quand même appuyer sur un bouton pendant des heures et puis [rires] mais (...) ouais non par contre Final Fantasy c'est vraiment, c'est vraiment top quoi, même encore maintenant j'veux dire avec l'histoire, l'univers et tout (Malik, H, 29 ans, employé de banque).

Chai (H, 26 ans, technicien), à l'inverse, considère les jeux FPS de valeur supérieure aux jeux consistant à faire progresser un avatar, parce que les jeux FPS font appel au «skill », ce qui désigne un ensemble de capacités apprises et entraînées, comprenant l'agilité dans le maniement du personnage, la précision de la visée, les compétences tactiques, tous centraux pour gagner dans ce jeu. Cette distinction correspond à une forme de légitimation récurrente qui mérite d'être détaillée davantage, celle des «compétences transférables ».

\section{Justifier l'engagement vidéoludique : valorisation des impacts du jeu hors du cadre ludique}

Des formes plus affirmatives de légitimation, sans pour autant que les enquêtés ne l'explicitent comme telle, consistent à revendiquer certains effets et composantes de la pratique ludique qui ont une valeur socialement reconnue indépendamment du jeu luimême. La première forme est l'argument de la création de liens sociaux, ou l'entretien de 
relations existantes. C'est ce qu'avancent surtout les joueurs de jeux en ligne. Il peut s'agir de l'établissement de liens avec des personnes qui deviennent amis, confidents ou encore des relations amoureuses.

Y a plein de choses qui se sont passées depuis que j'ai commencé [le jeu FPS] hein.

J'ai rencontré une copine aussi sur le jeu (Melek, H, 20 ans, webdesigner indépendant).

Lichens ( $\mathrm{H}, 51$ ans, médecin) valorise le fait d'entretenir des relations « avec des gens de toute l'Europe » et constate que ces relations devenues pour une part des amitiés sont la raison principale du maintien de sa pratique. Il raconte aussi que ces relations peuvent s'étoffer par une rencontre organisée lors de vacances faites dans le pays en question, rejoignant par-là d'autres comme Aliss (F, 30 ans, informaticienne de gestion) qui a rencontré son meilleur ami par un jeu de rôle sur Internet six ans auparavant. Beaucoup de rencontres en ligne restent au niveau de simples "connaissances". Mais même lorsque la relation reste confinée au monde du jeu et qu'on ne sait pas grand-chose de son partenaire de jeu $^{16}$, ces liens faibles peuvent contribuer à justifier l'engagement vidéoludique. Kriek (H, 28 ans, employé de banque) souligne que les rencontres sont secondaires dans le sens où elles ne constituent pas la raison principale mais qu'elles sont « un moyen finalement d'avoir une vie, un aspect de vie sociale sur internet ».

Une seconde forme de valorisation d'éléments découlant la pratique vidéoludique sont les dispositions ou "compétences transférables» qu'identifient les joueurs. Cette légitimation consiste précisément à souligner les savoirs et apprentissages que la pratique du jeu a favorisés ou requis. Les exemples les plus évidents se trouvent dans l'acquisition de compétences et connaissances enseignées dans des institutions scolaires, comme l'entraînement à une langue en rejoignant une équipe internationale ainsi qu'avec des compétences moins institutionnelles, par exemple les capacités de gestion d'équipe :

Et en plus c'était européen donc je devais parler anglais, (...) c'était vraiment intéressant parce que cette langue, depuis que j'y joue je me suis vraiment amélioré en anglais aussi (Melek, H, 20 ans, webdesigner indépendant).

Mais c'est vrai que bon j'ai quand même une certaine expérience en team et tout ça, en termes d'organisation. J'pense que ça peut être pas mal mais il faut pas transposer ça dans le monde professionnel directement, faut peut-être se servir de quelques petites choses quoi (Onyx, $\mathrm{H}, 27$ ans, étudiant en droit).

47 Les autres joueurs dont l'engagement vidéoludique comportait des tâches organisationnelles et de communication font des rapprochements similaires, mentionnant parfois le monde du travail. Pour Aliss (F, 30 ans, informaticienne de gestion), il y a une continuité entre le rôle de meneuse qu'elle finit toujours par adopter dans le jeu et son sens de l'initiative et de l'organisation qu'elle adopte spontanément au bureau.

Plus concrètement encore, la pratique $\mathrm{du}$ jeu vidéo peut avoir des répercussions professionnelles en termes de compétences en informatique apprises en jouant ou, plus précisément, apprises afin de pouvoir jouer. En effet, les joueurs ayant pratiqué les jeux sur des supports comme les ordinateurs Amiga, Commodore, les premiers PC sur DOS dans les années 1980 et 1990, racontent avoir dû comprendre le fonctionnement des systèmes d'exploitation, la configuration du hardware et parfois même de la programmation ; tout ceci simplement pour pouvoir installer et faire fonctionner les jeux. Plusieurs informaticiens interrogés disent avoir découvert ou pris goût à l'informatique de cette façon et remarquent que l'association est fréquemment faite par d'autres informaticiens. Cependant, ceci est propre au jeu sur PC, les jeux sur consoles nécessitant 
généralement moins de manipulations et connaissances logicielles ou matérielles. De plus les modalités du jeu sur PC ont évolué avec les technologies. Pour les générations plus récentes, les obstacles techniques au jeu ne semblent plus pousser à l'acquisition des mêmes compétences, mais d'autres enjeux apparaissent comme la gestion d'un site web, d'un forum, des médias "sociaux ». Compétences dont l'acquisition est par exemple racontée par Melek, pour qui elles sont devenues une raison de se lancer en tant qu'indépendant dans le webdesign comme activité professionnelle à la place de sa formation de monteur-électricien prévue :

J'ai décidé de créer ma propre team en fait. Et c'est vraiment à ce moment-là que j'ai dû faire un site web pour mon équipe, et j'ai vraiment découvert ce domaine à ce moment-là et ça m'a vraiment vraiment passionné quoi (Melek, H, 20 ans, webdesigner indépendant).

Les efforts de banalisation du jeu vidéo détaillés plus haut se prolongent aussi dans l'analogie avec la pratique du sport d'équipe en club. Comme le raconte Chai $(\mathrm{H}, 26$ ans, technicien), sensible à la question puisqu'il pratique le football en club, le jeu FPS en ligne lui a enseigné de la maturité : « sur tout, dans le respect du joueur d'en face, d'apprendre à fermer sa bouche et moins narguer les gens en fait ». Un enquêté (Ork, H, 38 ans, technicien), explique que pour devenir l'entraîneur d'une équipe de hockey locale, il avait tablé sur son expérience de gestion d'équipes dans un jeu de tir lors d'un entretien avec les responsables du club, puisqu'il avait certes de l'expérience comme joueur de hockey mais pas encore en tant qu'entraîneur dans ce cadre-là. Il apparaît que ce sont surtout les joueurs ayant réussi à rendre leur pratique socialement acceptable en trouvant un " équilibre » grâce à l'autocontrôle acquis au fil de leur expérience, qui peuvent accéder à ces formes de légitimation. Formes qui consistent à valoriser les relations, les dispositions et les connaissances apprises au contact du jeu, à reconnaître en somme la dimension socialisatrice que peut avoir le jeu vidéo comme toute autre activité en société.

\section{Un projet de transformation des valeurs : le cas du e-sport}

50 Les formes de légitimation que mobilisent les joueurs sont pour une grande partie implicites et peu revendicatives, tout comme les manifestations d'illégitimité et d'inacceptabilité sont généralement peu tangibles et relèvent davantage du domaine de l'anticipation. Mais les joueurs ont parfois des positions tranchantes, comme pour Sirius qui en livre une lecture teintée de sociologie :

C'est difficile de le faire comprendre en général t'en parles pas trop parce que malheureusement, je prends mon exemple, (...) ma mère et mon père sont des personnes qui sont entre guillemets un peu... comment expliquer ça, bourgeoises quoi. Ma mère est directrice artistique mon père est directeur de toute la publicité pour un journal, donc euh, ce genre de choses-là pour eux c'est futile, tu vois ? Ils se disent c'est un jeu quoi on en a rien à foutre (Sirius, H, 20 ans, BTS en emploi).

51 Sirius situe la dévalorisation du jeu vidéo par ses parents en soulignant leur milieu social, et il identifie par ailleurs le fait que «les jeunes générations comprennent assez rapidement » contrairement à la génération de ses parents. Partant de ce constat, il décrit comment il souhaite faire évoluer les "mentalités " pour faire mieux reconnaître la valeur des jeux vidéo. Ce qui nous amène à l'exemple des joueurs qui mettent en avant l'idée du e-sport (pour «sport électronique » en anglais), une conception du jeu vidéo fonctionnant comme un sport à part entière (Mora \& Héas, 2003) : 
Vraiment on arrive à un niveau où ça devient professionnel et la seule chose qui manque en gros c'est que les gens arrêtent de penser que les jeux vidéo c'est 'tu passes du temps pour rien' c'est pas... il y a un jeu, sauf que du coup forcément dans tout jeu il y a de la compétition. Tu vois le football c'est un jeu hein, pourtant t'as ceux qui sont très bons au football et qui plaisent à ceux qui jouent un peu au football ils aiment bien voir ceux qui savent vraiment ce que c'est que de tirer dans un ballon. Là c'est pareil. (...) Si demain on en montrait à la télé ben pour eux ça deviendrait logique que c'est un sport (Sirius, H, 20 ans, BTS en emploi). vouent les joueurs en tant que spectacle. Il s'engage dans les jeux comme joueur et surtout en tant qu'entraîneur et manager, et espère pouvoir en faire une profession un jour. La forme de légitimation ici cumule certaines des formes précédentes, car elle propose que le jeu vidéo soit considéré comme faisant partie d'une catégorie plus établie, que ses impacts au-dehors soient reconnus, mais elle est plus ambitieuse que la banalisation du jeu discutée plus tôt. On pourrait parler de l'émergence d'une identité «projet » au sens de Castells (1997). Il s'agit effectivement d'un projet de transformation des représentations courantes du jeu par la création d'associations promouvant le sport électronique et leur établissement dans un paysage médiatique et économique en s'associant à des sponsors (fabricants de composants, chaînes de magasins multimédia, fournisseurs de services Internet et médiatiques,...). Sirius et ses collègues ont ainsi pour projet collectif ${ }^{17}$ de rendre la société attentive au potentiel sportif du jeu vidéo. D'autres joueurs, sans être engagés dans l'organisation de compétitions ou la promotion des jeux voient dans l'analogie avec le sport des manières de valoriser leur pratique. Kriek par exemple, a participé à des compétitions de Tetris : « Au Tetris World Championship, donc la Coupe Davis du Tetris, j'étais dans l'équipe », et raconte sa fierté d'avoir été membre d'une équipe réputée : «y avait des équipes vraiment comme quand tu vois au foot Barcelone Real de Madrid, et moi j'ai été recruté dans une des plus grandes équipes qui existait depuis 1997, donc ça c'était l'apothéose de ma carrière " (Kriek, H, 28 ans, employé de banque). Même si ces analogies sont faites avec des intonations indiquant une dose d'humour pour montrer que l'interviewé a conscience de la différence considérable en termes de reconnaissance entre les compétitions vidéoludiques citées et les institutions sportives de grande envergure, les expériences sont sources de fierté et contribuent à valoriser son engagement vidéoludique.

\section{Conclusion}

Il semble aujourd'hui que le jeu vidéo a acquis davantage de légitimité, notamment de par un processus générationnel que relève Berry (2012) : les joueurs sont davantage que dans les années 1980-1990 en mesure de défendre la légitimité de leur loisir notamment de par leur statut d'adulte; ceci s'inscrit dans un processus plus englobant de valorisation de ce qui est couramment nommé la culture geek, « au travers de livres, de thèses ou de séries télévisées dans lesquels la figure du geek passionné par la science-fiction et l'informatique n'est plus seulement l'objet de moquerie mais une figure centrale» (Berry, 2012: 45). Mais le fait que le jeu vidéo soit reconnu de plus en plus en tant que composante culturelle ordinaire des sociétés contemporaines ne signifie pas que les divers types de jeux soient devenus culturellement légitimes, ni que les façons de les pratiquer soient d'emblée acceptables. 

sinon légitime, au moins digne d'intérêt. Mais dans leurs pratiques ordinaires, les joueurs eux-mêmes sont moins préoccupés par la légitimité culturelle du jeu vidéo que par la gestion de l'acceptabilité des modalités de leur pratique au regard d'autrui et par rapport à leurs évaluations du temps bien investi. C'est donc dans les formes d'engagement que se situe le problème de la bonne "place» du jeu telle que perçue par les joueurs et leurs entourages. Nos enquêtés semblent en premier lieu souhaiter que leur propre façon de s'engager dans le jeu vidéo soit considérée comme un loisir ordinaire, banal, " pas pire » que d'autres. Un cadrage du jeu vidéo fréquemment proposé par les joueurs est celui du sport, tant comme activité d'amateur que comme spectacle. Les joueurs participant à l'élaboration de projets visant une légitimation d'envergure plus sociétale, eux aussi, ont moins à cœur de faire considérer le jeu comme une forme d'art que comme une forme de «sport ", notamment en tant qu'activité lucrative. La légitimation du jeu comme forme d'art, qui semble être davantage un projet propre au monde des concepteurs de jeux vidéo, trouve peut-être son pendant dans les qualifications que les joueurs utilisent pour hiérarchiser leurs goûts vidéoludiques, ce qui constitue une piste d'approfondissement pour nos recherches ultérieures. sociale de leur loisir. Au quotidien, c'est l'inacceptabilité des façons de jouer, en particulier concernant les modalités temporelles de la pratique qui préoccupe principalement les joueurs. Ces jugements proviennent la plupart du temps de l'entourage proche, en particulier les parents et le conjoint ; mais il apparaît aussi que les joueurs intériorisent et reproduisent des normes sur les jeux et pratiques acceptables. Lorsqu'aux modalités de la pratique s'ajoutent des jugements négatifs sur la nature du jeu, les joueurs sont plus démunis ; dans les deux carrières de joueurs ayant débouché sur un arrêt de la pratique vidéoludique (Gauthier et Malik), cette conjonction entre inacceptabilité sociale et illégitimité culturelle est observable.

joueurs qui parviennent à maintenir une pratique vidéoludique le font en anticipant les jugements d'inacceptabilité, en trouvant des manières de valoriser le jeu et en adaptant les modalités de leur pratique. Par exemple, le fait que les joueurs tiennent souvent à définir leur jeu comme « une activité parmi d'autres » indique que le jeu vidéo est acceptable à condition de ne pas être exclusive. Ces cadrages de la « bonne " pratique s'inscrivent dans des conceptions normatives concernant tant la gestion des loisirs que des valeurs plus générales, par exemple sur le couple, la famille ou l'amitié, et dans les attentes de rôle qui en découlent. Etant donné ces attentes normatives auxquels souscrivent en principe les joueurs, même très engagés, le développement d'un autocontrôle s'avère nécessaire pour concilier l'engagement vidéoludique avec les attentes d'autrui.

Ces considérations sur les logiques de légitimation donnent lieu à des pistes d'approfondissement qui nous semblent intéressantes. Les joueurs ne sont pas égaux face aux conditions de légitimité et moyens de légitimation. L'étape de vie dans laquelle ils se trouvent paraît importante à cet égard: elle est constituée de rôles sociaux (enfant, adulte, parent, etc.) donnant lieu à des attentes spécifiques en termes de gestion du temps de loisir et de choix des activités, ainsi qu'à des ressources en termes de statut, d'autonomie, de ressources économiques etc. Certains joueurs disposent de plus d'autonomie que d'autres pour légitimer leur engagement dans le jeu: adulte et au bénéfice d'un métier qu'il valorise, Samuel ( $\mathrm{H}, 37$ ans, informaticien) a moins de

RESET, 4 | 2015 
problèmes que Gauthier (20 ans, étudiant de bachelor) à considérer que c'est un « hobby comme un autre pour notre génération ». En même temps, Samuel, sur le point de devenir père, sait qu'il ne peut pas se permettre de maintenir un engagement dans le jeu comme administrateur, alors que Sirius ( $\mathrm{H}, 20$ ans, BTS en emploi) peut espérer convaincre ses parents que le jeu vidéo est à même de devenir un sport reconnu en continuant de développer son engagement en tant que manager. Ceci amène un second élément d'approfondissement: en plus des conditions d'acceptabilité changeantes avec les étapes de vie, les pratiques vidéoludiques soutenues dans le long terme peuvent constituer des sources de légitimité. Pour qu'un joueur puisse être reconnu à l'extérieur du jeu pour sa participation à un équivalent vidéoludique de la Coupe Davis ou pour sa connaissance approfondie de la fiction d'un monde de jeu de rôle, il faut encore qu'il ait acquis ces expériences et des occasions de les faire valoir. Ainsi, outre les logiques de hiérarchisation opérées par les joueurs, la comparaison détaillée des étapes de vie ainsi que ce qu'on pourrait appeler le " capital vidéoludique » apparaissent comme des objets à étudier plus avant.

\section{BIBLIOGRAPHIE}

AARSETH Espen (2007). «I fought the law: Transgressive play and the implied player ». Situated Play. Proc. DiGRA, 24-28.

ANDERSON Craig A., \& BUSHMAN Brad J. (2001). «Effects of violent video games on aggressive behavior, aggressive cognition, aggressive affect, physiological arousal, and prosocial behavior: A meta-analytic review of the scientific literature », Psychological science 12 (5), pp. 353-359.

BAMBERG Michael GW. (1997). « Positioning between structure and performance », Journal of narrative and life history 7 (1-4), pp. 335-342.

BERRY Vincent (2008). « Les cadres de l'expérience virtuelle : analyse de l'activité ludique dans les MMO » UQTR: OMNSH [en ligne], consulté 10.10.2015. URL : http://www.omnsh.org/spip.php? page=imprimer\&id_article $=145$.

BERRY Vincent (2012). L'expérience virtuelle: jouer, vivre, apprendre dans un jeu vidéo, Rennes, Presses universitaires de Rennes.

Berry Vincent, CoAvoux Samuel \& Boutet Manuel (2014). « Playing Styles. The Differentiation of Practices in Online Video Games ", in Grenfell Michael \& Lebaron Frédéric (dir.) Bourdieu and Data Analysis. Methodological Principles and Practice, Bern, Peter Lang, pp.165-180.

BoURDIEU Pierre (1979). La Distinction: Critique Sociale Du Jugement, Paris, Éditions de Minuit.

BoutET Manuel (2008). «S'orienter dans les espaces sociaux en ligne. L'exemple d'un jeu », Sociologie du Travail 50 (4), pp. 447-470.

BoutET Manuel (2012). « Jouer aux jeux vidéo avec style. Pour une ethnographie des sociabilités vidéoludiques ». Réseaux, 3 (173-174), pp. 207-234. 
CASTELLS Manuel (1997). The power of identity: The information age: Economy, Society, and Culture Vol II, Londres, Wiley-Blackwell.

CoHEN Stanley (1980). Folk Devils and Moral Panics: The Creation of the Mods and Rockers, Londres/ New-York, Routledge.

COVER Rob (2006). « Gaming (ad) diction: discourse, identity, time and play in the production of the gamer addiction myth », Game Studies 6 (1), pp. 15.

CUnNingham Scott, EngelSTÄTTER Benjamin \& WARD Michael R. (2011). « Understanding the Effects of Violent Video Games on Violent Crime ", ZEW Discussion Papers, 11-042 [en ligne], consulté 10.10.2015. URL : http://papers.ssrn.com/sol3/papers.cfm?abstract_id=1804959\#.

DAJEZ Frédéric \& Roucous Nathalie (2009a). « Montre-moi tes jeux vidéo ! », in Delalande Julie (dir.) Des enfants entre eux. Des jeux, des règles, des secrets, Paris, Autrement, pp. 74-91.

DAJEz Frédéric \& Nathalie Roucous (2009b). Culture enfantine et ludiciels, Paris, Ministère de la Culture et de la Communication.

DeLlWING Michael (2013). «Addiction Diagnoses as Involvement Controls: The Pragmatic Value of Psychiatric Definitions of the Situation », RESET. Recherches en sciences sociales sur Internet, 1 (2) [en ligne], mis en ligne 01.01.2012, consulté 10.10.2105. URL : http://www.journal-reset.org/ index.php/RESET/article/view/17.

FERGUSON Christopher (2007). « The good, the bad and the ugly: A meta-analytic review of positive and negative effects of violent video games ", Psychiatric Quarterly 78 (4), pp. 309-316.

FERGUSON Christopher (2008). «The School Shooting/Violent Video Game Link: Causal Relationship or Moral Panic? » Journal of Investigative Psychology and Offender Profiling 5 (1-2), pp. 25-37.

FINE Gary Alan (1983). Shared fantasy: Role playing games as social worlds, Chicago, University of Chicago Press.

GENTILE Douglas, CHOO Hyekyung, LIAU Albert, SIM Timothy, LI Dongdong, FUnG Daniel \& KHOO Angeline (2011). « Pathological Video Game Use Among Youths: A Two-Year Longitudinal Study », Pediatrics, 127 (2), pp. 319-329.

GERBER David (2010). « Succès, dangerosité et illégitimité. Les représentations du jeu numérique dans les magazines d'actualité suisses depuis 2004 », in Craipeau Sylvie, Genvo Sébastien \& Simonnot Brigitte (dir.) Les jeux vidéo au croisement du social, de l'art et de la culture.

GOFFMAN Erving (1975). Stigmate: les usages sociaux des handicaps, Paris, Éditions de Minuit. GOFFMAN Erving (1986). Frame Analysis: An Essay on the Organization of Experience, Lebanon, Northeastern University Press.

GRANIC Isabela, LOBEL Adam \& C.M.E ENGELS Rutger (2013). « The benefits of playing video games », American Psychologist, 69 (1), pp. 66-78.

GREEN C. Shawn \& BAVELIER Daphne (2003). « Action video game modifies visual selective attention », Nature, 423 (6939), pp. 534-537.

JENKINS Henry (2006). Fans, bloggers, and gamers: exploring participatory culture, New York, New York University Press.

JOFFE Hélène (1999). Risk and «the Other », Cambridge, Cambridge University Press.

JOUËT Josiane (2000). « Retour critique sur la sociologie des usages », Réseaux 18 (100), pp. 487-521. 
KLEIBER Douglas A. \& KELLY John R. (1980). « Leisure, socialization, and the life cycle », in Iso-Ahola Seppo E (dir.) Social psychological perspectives on leisure and recreation, Springfield, Charles C Thomas, pp. 91-138.

LAHIRE Bernard (2004). La culture des individus. Dissonances culturelles et distinction de soi, Paris, La Découverte.

LAHIRE Bernard (2011). « Distinctions Culturelles et Lutte de Soi Contre Soi : 'Détester La Part Populaire de Soi' », Hermès, 2 (42), pp. 137-143.

MAUCo Olivier (2011). «L'institutionnalisation de la violence des jeux vidéo à l'Assemblée nationale », Quaderni, 75, pp. 51-62.

MORA Philippe \& Stéphane HÉAS (2003). « Du joueur de jeux vidéo à l'e-sportif: vers un professionnalisme florissant de l'élite? », in Roustan Mélanie (dir.) La pratique du jeu vidéo : réalité ou virtualité, Paris, L'Harmattan, pp. 132-146.

NARDI Bonnie (2010). My Life as a Night Elf Priest: An Anthropological Account of World of Warcraft, Ann Arbor, University of Michigan Press.

NeUgarten Bernice L., Moore Joan W. \& lowe John C. (1965). « Age Norms, Age Constraints, and Adult Socialization », American Journal of Sociology, 70 (6), pp. 710-717.

PASQUIER Dominique (2005). Cultures lycéennes: la tyrannie de la majorité, Paris, Autrement. PASQUIER Dominique (2010). «Culture sentimentale et jeux vidéo : le renforcement des identités de sexe ", Ethnologie française, 40 (1), pp. 93-100.

QUANDT Thorsten, GRUENINGER Helmut \& WIMMER Jeffrey (2009). « The Gray Haired Gaming Generation: Findings From an Explorative Interview Study on Older Computer Gamers », Games and Culture, 4 (1), pp. 27-46.

RAYMORE Leslie A., BARBER Bonnie L., ECCLEs Jacquelynne S. \& GODBEY Geoffrey C. (1999). « Leisure behavior pattern stability during the transition from adolescence to young adulthood ", Journal of Youth and Adolescence, 28 (1), pp. 79-103.

RUEFF Julien (2008). « Où en sont les "game studies" ? ", Réseaux, 5 (151), pp. 139-166.

SHAPIRO Roberta \& HEINICH Nathalie (2012). «When is Artification? », Contemporary Aesthetics, 4 [en ligne], consulté 11.10.2015. URL : http://hdl.handle.net/2027/spo.7523862.spec.409.

SMUTS Aaron (2005). « Are Video Games Art? », Contemporary Aesthetics, 3 [en ligne], consulté 11.10.2015. URL : http://www.contempaesthetics.org/newvolume/pages/article.php? articleID $=299$.

SWEETSER Penelope \& Peta WYETH (2005). « GameFlow: a model for evaluating player enjoyment in games », Computers in Entertainment (CIE), 3 (3), pp. 33.

TAYLOR T. L. (2006). Play between worlds: Exploring online game culture, Cambridge, MIT Press.

TER MINASSIAN Hovig (2013). « Les jeux vidéo. Un patrimoine culturel ? », Géographie et cultures, 82 [en ligne], mis en ligne 22.02.2013, consulté 22.12.2015. URL : http://gc.revues.org/1393 ; DOI : $10.4000 /$ gc. 1393 .

THORnHAM H. (2009). «Claiming a Stake in the Videogame », Convergence: The International Journal of Research into New Media Technologies, 15 (2), pp. 141-159. 
TRICLOT Mathieu (2013). «Game studies ou études du play? Une lecture croisée de Jacques Henriot et de Jesper Juul », Sciences du jeu, 1 [en ligne], mis en ligne 01.10.2013, consulté 10.10.2015. URL / http://sdj.revues.org/223 ; DOI : 10.4000/sdj.223.

VORDERER Peter, HARTMANN Tilo \& KLIMMT Christoph (2003). « Explaining the enjoyment of playing video games: the role of competition ", Proceedings of the second international conference on Entertainment computing, Pittsburgh, Carnegie Mellon University [en ligne], consulté 15.11.2015. URL : http://portal.acm.org/citation.cfm?id=958735.

WENDLING Thierry (2006). « Une approche anthropologique de l'addiction aux jeux vidéo », Dépendances, 28, pp. 21-24.

Williams Dmitri (2003). « The video game lightning rod ", Information Communication \& Society, 6 (4), pp. 523-550.

WILLIAMS Dmitri, YeE Nick \& CAPLAN Scott E. (2008). « Who plays, how much, and why? Debunking the stereotypical gamer profile ", Journal of Computer-Mediated Communication, 13 (4), pp. 993-1018. WOLF Mark J. P. (2012). « Videogames as an Artform », in Wolf Mark J. P. (dir.), Encyclopedia of Video Games: The Culture, Technology, and Art of Gaming, Greenwood, ABC-CLIO.

YEE Nick (2006). « Motivations for Play in Online Games », CyberPsychology \& Behavior 9 (6), pp. 772-775.

YouNG Kimberly S. (1998). « Internet addiction: The emergence of a new clinical disorder ». CyberPsychology \& Behavior 1 (3), pp. 237-244.

ZABBAN Vinciane (2012). « Retour sur les game studies. Comprendre et dépasser les approches formelles et culturelles du jeu vidéo », Réseaux, 3 (173-174), pp. 137-176.

\section{NOTES}

1. Le Museum of Modern Art de New York expose par exemple depuis 2013 des jeux vidéo dans sa collection permanente.

2. Si N. Heinich et R. Shapiro insistent sur le fait qu'il ne faut pas confondre artification et légitimation, elles soulignent tout de même qu'il s'agit d'un processus qui précède celui de l'accès éventuel à la légitimité.

3. Woitier, Chloé. «Au Grand Palais, le jeu vidéo atteint enfin la consécration ». Le Figaro, 10 novembre 2011 [en ligne], consulté 05.11.2015. URL : http://www.lefigaro.fr/ hightech/2011/11/10/01007-20111110ARTFIG00567-au-grand-palais-le-jeu-video-atteint-enfinla-consecration.php

4. Les game studies sont le champ pluridisciplinaire d'étude du jeu - surtout sous sa forme électronique - qui a émergé avec l'avènement des jeux vidéo des années 1900 et s'est progressivement autonomisé au début des années 2000 (voir à ce propos Zabban 2012 ; ou encore Rueff 2008). Les game studies combinent un intérêt "académique " pour le jeu avec un intérêt " appliqué », orienté vers la conception et l'utilisation du jeu vidéo à diverses fins, y compris des fins non-ludiques comme l'enseignement, la thérapie, etc.

5. Sheffield, Brandon. "Video games are a legitimate form of cultural expression... right?» Gamasutra, 22 avril 2013 [en ligne], consulté le 04.11.2015. URL : http://gamasutra.com/view/ news/190916/Video_games_are_a_legitimate_form_of_cultural_expression_right.php.

6. À titre d'exemple: Anderson, Dan. «The Gamer Stigma--Why the Hate? »1Up.com, 12 juillet 2011 [en ligne], consulté 05.11.2015. URL : http://www.1up.com/do/blogEntry?bId=9084243.

7. http://stigma-videospiele.de et http://www.gamerights.ch. 
8. Ceci s'explique par le fait que ces travaux sont souvent pensés dans une optique disciplinaire différente, psychologique par exemple (Vorderer, Hartmann, et Klimmt 2003), ou encore parce qu'ils sont orientés vers la conception et le développement de jeux (Sweetser \& Wyeth 2005).

9. Le recrutement a visé à faire varier les profils en termes de dispositifs de jeu (joueurs PC et consoles, joueurs de divers genres de jeux vidéo), de caractéristiques socio-démographiques (âges, professions, état civil...) et de « carrières » ludiques (joueurs ayant opté pour un emploi en lien avec le jeu vidéo, ex-joueurs...) mais il faut signaler que le population enquêtée est avant tout constituée de joueurs suffisamment investis dans leur pratique ludique pour participer dans les forums et "tchats» en ligne, ou du moins pour se reconnaitre une pratique ludique suffisamment significative pour accepter de faire un entretien. Les enquêtés ont été contactés à l'occasion de deux terrains ethnographiques dans des jeux en ligne, par recrutement en «boule de neige ", ainsi que via la passation d'un questionnaire dans trois classes de formation professionnelle en cours d'emploi à Genève.

10. Acronyme pour l'expression anglaise in real life. C'est un terme fréquemment employé par les joueurs pour désigner les événements qu'ils organisent en face-à-face.

11. FPS est l'acronyme pour first-person shooter, ou jeu de tir en vue subjective, un genre dont la pratique se perpétue depuis les titres PC des années 1990 comme Wolfenstein 3D, Doom ou Quake jusqu'aux Call of Duty et autres Battlefield figurant parmi les meilleures ventes console actuellement.

12. Il s'agit d'un titre appartenant au genre dit hack'n'slash, bien connu parmi les joueurs assidus et souvent considéré comme chronophage.

13. Voir à ce propos Lahire (2008).

14. Le trickjumping consiste, généralement dans les jeux de type FPS, à se déplacer agilement en usant du moteur physique du jeu, transformant ainsi le jeu de tir en jeu de parcours. Il s'agit typiquement une pratique «émergente» (Pearce 2006), non prévue initialement par les développeurs du jeu.

15. Le terme casual sert à qualifier des pratiques ludiques occasionnelles, de durée généralement brève et désigne parfois des joueurs peu engagés et peu experts : ceux que les enquêtés appellent les « casus ».

16. Une connaissance dans le jeu peut parfois être connue par son pseudonyme depuis des années sans que le nom légal n'ait besoin d'être divulgué.

17. Sirius se réfère aux acteurs de la scène $e$-sport, mus par le même projet, comme en témoignent les sites web d'équipes, ligues et autres protagonistes du e-sport.

\section{RÉSUMÉS}

Partant du constat que la pratique des jeux vidéo a un statut incertain en termes de valeur culturelle et d'acceptabilité sociale, cet article esquisse les manières dont les joueurs de jeux vidéo se confrontent à des contraintes normatives et négocient les conditions d'acceptabilité de leur pratique. L'analyse de récits biographiques de 28 joueurs aux carrières ludiques variées permet de décrire comment se manifeste l'inacceptabilité de la pratique du jeu vidéo, puis de dresser un inventaire des positionnements adoptés face à la menace de discrédit: du simple évitement d'un stigmate jusqu'à l'ambition de transformer la place du jeu vidéo dans la société. Les joueurs n'ont pas les mêmes ressources pour rendre leur pratique acceptable, et nos résultats 
soulignent le poids de valeurs familiales intériorisées, du capital culturel et vidéoludique, ainsi que l'importance particulière de la position du joueur dans le parcours de vie.

Based on the premise that the cultural and social status of video games is ambiguous, this paper studies how gamers face normative constraints and how they negotiate the acceptability of their gaming. Through 28 biographical interviews of gamers with various profiles, it first outlines how the unacceptability of gaming manifests itself to players. It then describes the ways in which players deal with such discreditability. They range from simply avoiding stigma to trying to change the place of video games in society at large. Players however have unequal means at their disposal. Our results indicate the role of internalized family values, of cultural capital (and of specific ludic capital), and most importantly of the individual's position in the life course.

\section{INDEX}

Mots-clés : jeux vidéo, stigmate, légitimité, biographie, parcours de vie

Keywords : digital games, stigma, cultural legitimacy, biography, life course

\section{AUTEUR}

DAVID GERBER

Université de Genève, Département de sociologie 